\title{
Research Paper \\ Determining the Prevalence of Sleep Disorder and Its Predictors Among Elderly Residents of Nursing Homes of Ahvaz City in 2017
}

\section{Shahab Papi ${ }^{1}$, Zeynab Karimi ${ }^{2}$, Gholamreza Ghaed Amini Harooni ${ }^{3}$, Azadeh Nazarpour ${ }^{4}{ }^{*}$ Parvin Shahry ${ }^{5}$}

1. Department of Health Education and Promotion, Faculty of Health, Tabriz University of Medical Sciences, Tabriz, Iran.

2. Department of Health Education and Promotion, School of Health, Qom University of Medical Sciences and Health Services, Qom, Iran

3. Social Welfare Management Research Center, University of Social Welfare and Rehabilitation Sciences, Tehran, Iran.

4. Department of Rehabilitation Management, Faculty of Rehabilitation, Ahvaz Jundishapur University of Medical Sciences, Ahvaz, Iran.

5. Social Determinants of Health Research Center, Faculty of Health, Ahvaz Jundishapur University of Medical Sciences, Ahvaz, Iran.

\section{(A) 0}

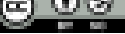

Received: 05 Sep 2018 Accepted: 29 Jan 2019 Available Online: 10 Mar 2019

Key words:

Elderly, Nursing home, Sleep disorder Concurrent disease, Social support citration Papi Sh, Karimi Z, Ghaed Amini Harooni Gh, Nazarpour A, Shahry P. [Determining The Prevalence of Sleep Disorder and Its Predictors Among Elderly Residents of Nursing Homes of Ahvaz City in 2017 (Persian)]. Salmand: Iranian Journal of Ageing. 2019; 13(Special Issue):576-587. https://doi.org/10.32598/SIJA.13.Special-Issue.576

https://doi.org/10.32598/SIJA.13.Special-Issue.576

\section{A B STRACT}

Objectives Sleep quality is an important indicator of many geriatric diseases. As people age, changes happen in the quality and quantity of their sleep, which means sleep disorders and complications. This study aimed to determine the predictors of sleep disorder in elderly people living in nursing homes.

Methods \& Materials This cross-sectional study was carried out on a sample of 130 elderly residents of senior care homes in Ahvaz City, Iran. The samples were selected by the convinience sampling method and according to inclusion and exclusion criteria. Pittsburgh sleep quality questionnaire, general health questionnaire (12 questions form), knee pain questionnaire, social support questionnaire, and demographic characteristic checklist were used to collect the study data that were analyzed using stepwise linear regression analysis in SPSS22.

Results In this study, 33.8\% ( $n=44)$ of the participants were men and $66.2 \%(n=86)$ were women. The Mean $\pm S D$ age of the subjects was $68 \pm 7.8$ years. In total, $13.8 \%$ of the samples had a low level of sleep disorder, $81.5 \%$ moderate level of sleep disorder, and $4.6 \%$ severe level of sleep disorder. Based on the fitted regression model $(F=7.24 ; P<0.001)$, multiple diseases $(\beta=0.20)$, perceived social support $(\beta=-0.26)$, joint stiffness $(\beta=0.25)$, and knee pain $(\beta=0.24)$ were the significant predictors of sleep disorder in the elderly people, and explained $18.9 \%$ of the changes in sleep quality.

Conclusion The results of the study showed that $86.1 \%$ of the elderly had a sleep disorder. Perceived social support, joint stiffness, knee pain, and multiple diseases were among determinants and predictors of elderly sleep disorder.

\section{Extended Abstract}

\section{Objectives}

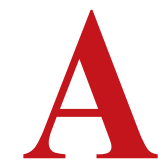

ging causes different physical and mental health problems and diseases in the elderly [1]. One of these problems is sleep disorder [2] such that about 50\% of older people complain about the difficulty in initiating or maintaining sleep [3]. Accordingly, based on studies, sleep disorders in nursing homes is more than $75 \%[4,5]$. Hence, there is a need to recognize the various aspects of sleep disorders in the elderly and enrich our knowledge about its affecting factors to better evaluate the problems associated with sleep disorders and to take the necessary measures to reduce it. Since few

\section{* Corresponding Author:}

Parvin Shahry, PhD.

Address: Social Determinants of Health Research Center, Faculty of Health, Ahvaz Jundishapur University of Medical Sciences, Ahvaz, Iran. Tel: +98 (61) 33738269

E-mail: shahry_2001@yahoo.com 
studies have been conducted to assess the predictors of sleep disorders in the elderly, especially those living in nursing homes, this study aimed to investigate the prevalence of sleep disorders and its predictors among elderly residents of nursing homes in Ahvaz City, Iran.

\section{Methods \& Materials}

This is a cross-sectional study conducted in 2017 . The statistical population consisted of all elderlies $(n=205)$ living in 4 nursing homes located in Ahvaz City, Iran. Using convenience sampling method and based on inclusion and exclusion criteria, 130 elderlies were selected as study samples. The inclusion criteria were ability to speak, willingness to participate in the study, aged older than 60 years, no cognitive disorders (i.e. memory impairment, dementia, Alzheimer) according to the medical records, and no participation in other studies at the same time. Those unwilling to participate were excluded from the study. Before data collection, written informed consent was obtained from each participant.

The study was also approved by the Research Ethics Committee of Ahvaz University of Medical Sciences (code: IR.AJUMS.REC1394.142). For collecting data, the Pittsburgh sleep quality index (PSQI), 12-item general health questionnaire (GHQ-12), VAUX social support questionnaire, Western Ontario and McMaster universities arthritis index (WOMAC), and a demographic form were used. After collecting data, they were analyzed in SPSS v.22. To present the variables, descriptive statistics such as mean and standard deviation were used, and for examining the concurrent effect of each predictor on elderly sleep disorder, stepwise linear regression analysis was carried out. The significance level was set at $0.05(\mathrm{P}<0.05)$.

\section{Results}

Of 130 participants, $86(66.2 \%)$ were men and 44 $(33.8 \%)$ women with a Mean \pm SD age of $68 \pm 7.8$ years. Regarding WOMAC score of the participants, their Mean \pm SD knee pain was $18.13 \pm 10.11$, while the Mean \pm SD joint stiffness was $4.13 \pm 2.18$ and Mean \pm SD physical function was $33.33 \pm 15.19$. Moreover, their Mean \pm SD perceived social support and general health were $25.24 \pm 7.72$ and $9.26 \pm 1.7$, respectively.

Regarding the evaluation of sleep quality of the participants, 18 (13.8\%) reported mild sleep disorders, $106(81.5 \%)$ moderate, and $6(4.6 \%)$ severe sleep disorders. The stepwise linear regression analysis results (Table 1) showed that "concurrent disease" $(\beta=0.2)$, "joint stiffness" $(\beta=0.259)$, and "knee pain" $(\beta=0.244)$ had a significant positive correlation with sleep disorder in subjects, while "perceived social support" was negatively correlated with having sleep disorders $(\beta=-$ 0.266). According to the coefficient of determination $\left(\mathrm{R}^{2}\right), 18.9 \%$ of changes in sleep disorders is predicted by the four above mentioned variables. The regression model has a good fit $(\mathrm{F}=7.24, \mathrm{P}<0.001)$.

\section{Conclusion}

According to the results, $86.1 \%$ of elders had sleep disorders. The perceived social support, concurrent disease, joint stiffness, and knee pain were predictors of sleep disorders in the elderly. They could predict $18.9 \%$ of sleep disorders. Further studies should be conducted to identify other factors affecting sleep disorders in older people. Furthermore, it is necessary to study these factors in more details, and some interventions should be conducted to reduce the impact of these factors.

Table 1. Regression analysis results

\begin{tabular}{cccccc}
\hline Predictor & B & SE & $\boldsymbol{B}$ & t & P \\
\hline (Constant) & 10.76 & 1.85 & - & 5.82 & 0.001 \\
\hline Concurrent disease & 0.247 & 0.109 & 0.200 & 2.27 & 0.025 \\
\hline Perceived social support & -0.125 & 0.040 & -0.266 & 3.08 & 0.003 \\
\hline Joint stiffness & 0.433 & 0.160 & 0.259 & 2.71 & 0.008 \\
\hline Knee pain & 0.086 & 0.034 & 0.244 & 2.52 & 0.013 \\
\hline
\end{tabular}

$\mathrm{R}=0.435 ; \mathrm{R}^{2}=0.189 ; \mathrm{F}=7.24 ; \mathrm{P}<0.001$ 


\section{Ethical Considerations}

Compliance with ethical guidelines

This study has been approved by Research Ethics Committee of Ahvaz Jundishapur University of Medical Sciences (Code: IR.AJUI.REC.1394.142).

\section{Funding}

This study has been approved by Research Deputy of Ahvaz Jundishapur University of Medical Sciences (code: $77 \mathrm{~s} 93$ ).

\section{Authors' contributions}

Conceptualization, methodology and validation: Shahab Papi, Zeynab Karimi, Parvin Shahri; Investigation and formal analysis, resources, and draft preparation: Gholamreza Ghaed Amini Harooni, Shahab Papi, Parvin Shahri; Editing: Zeynab Karimi, Azadeh Nazarpour; and Supervision and project administration: Parvin Shahri.

\section{Conflict of interest}

The authors declared no conflict of interest.

\section{Acknowledgments}

The authors would like to thank authorities of Ahvaz Jundishapur University of Medical Sciences and Retired Organization of Ahvaz City and all elders that contributed in this article. 


\title{
بررسى شيوع اختلالات خواب و ييشبينى كنندهاى أن در سالمندان مقيم سراى سالمندان شهر

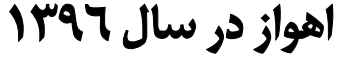

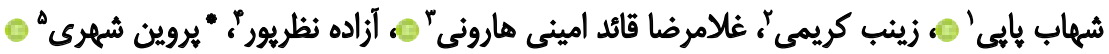 \\ 1- كروه آموزش بهداشت و ارتقاى سلامت، دانشكده بهيداشت، دانشكاه علوم يزشكى تبريز، تبريز، ايران.

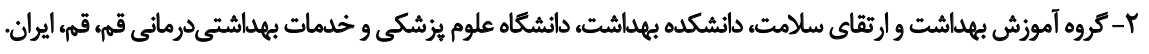

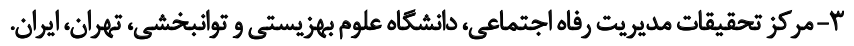

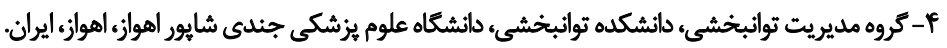

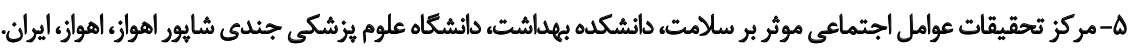

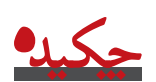

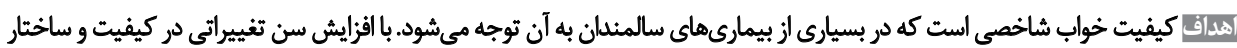

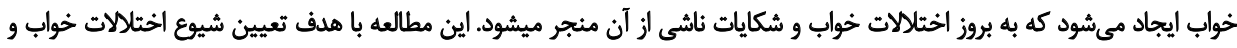

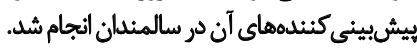

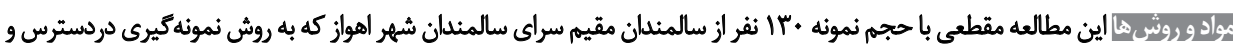

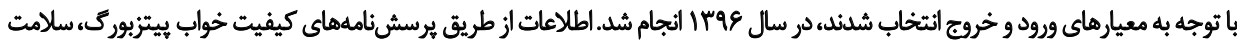

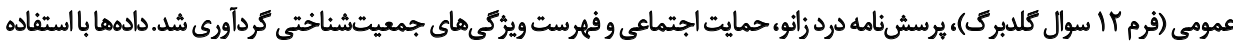

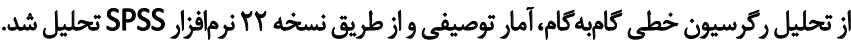

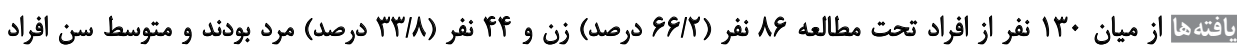

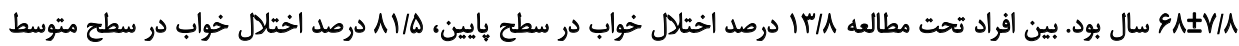

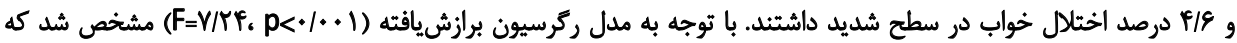

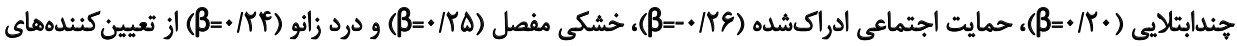

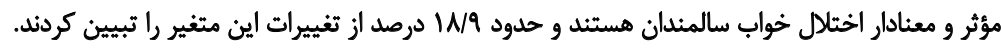

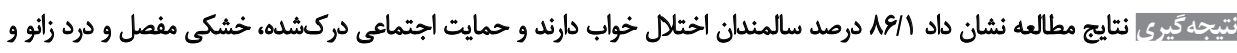
جيندابتلايى از تعيين كنئدهاي اختلال خواب سالمندان هستئد.
\end{abstract}

تاريخ دريافت: IF أشهريور

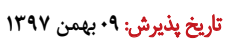
تاريخ انتشار: 19 اسفند 4 تون

از جمله مشكلات اين دوران، اختلالات مربوط به كيفيت

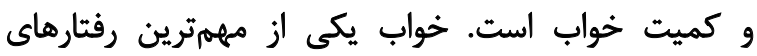

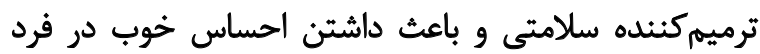

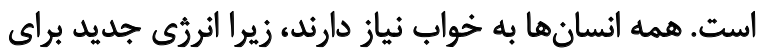

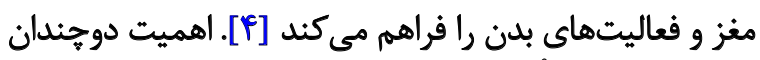

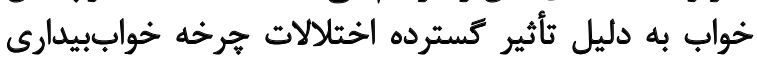

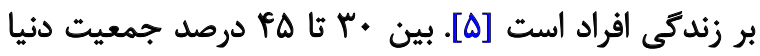

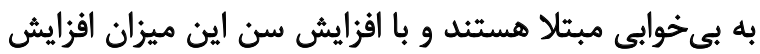

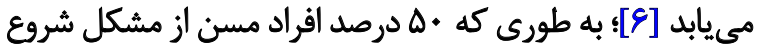

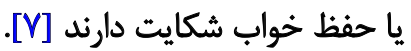

dato

ايران به عنوان يك كشور در حال توسعه، در آينده با جمعيت

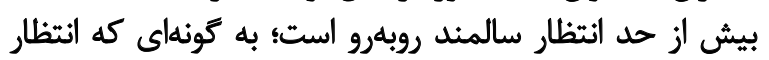

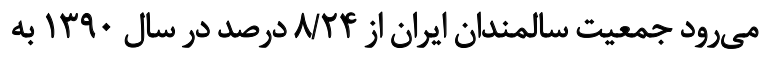

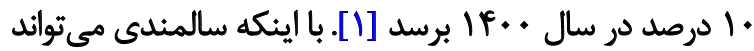

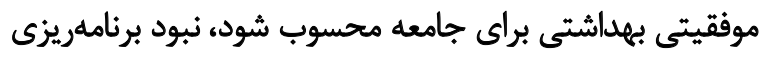

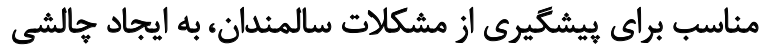

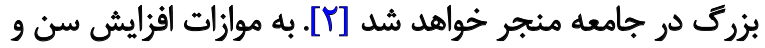

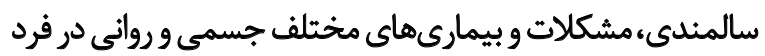

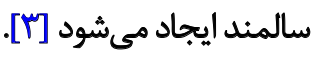
تلفن: يست الكترونيكي: shahry_2001@yahoo.com 
كرد و نسبت به رفع يا كاهش ميزان آن، خدمات لازم را ارائه

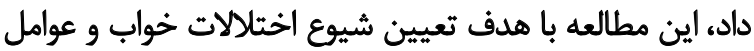

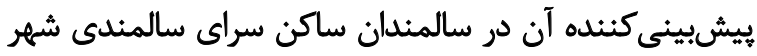

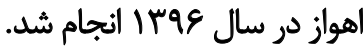

\section{ورشّ مطاُ}

اين يُروهش كه از نوع مطالعات مقطعى است با هدف تعيين

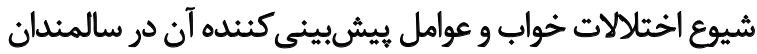

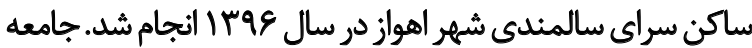

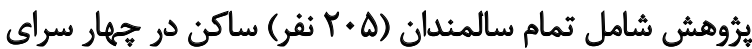

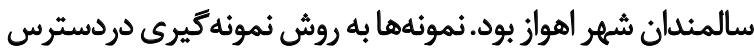

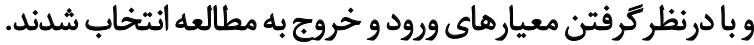

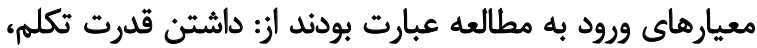

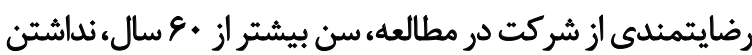

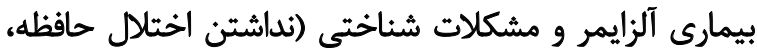
دمانس، آلزايمر) و حضورنداشتن در ديكر مطالعات برؤوهشى. به منظور قضاوت درباره مشكلات يادشده و نيز سوابق بيمارى

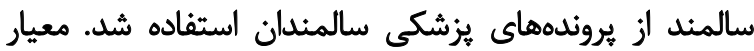

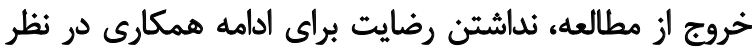

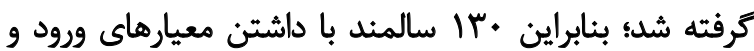

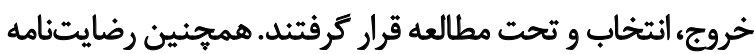

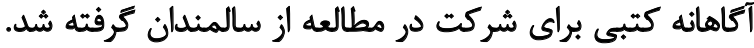

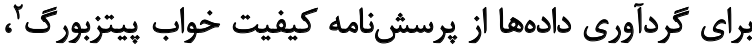

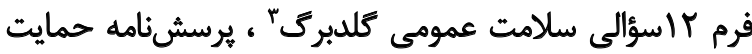

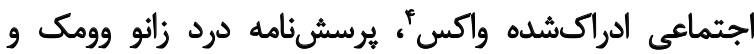
فهرست مشخصات جمعيتشناختى استفاده شد.

\section{مقياس كيفيت خواب يبئزبورك}

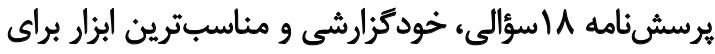

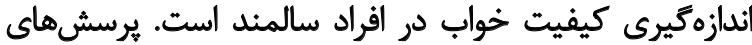

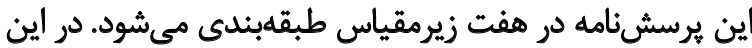

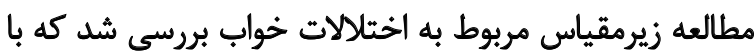

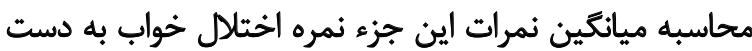

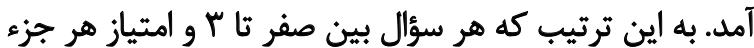

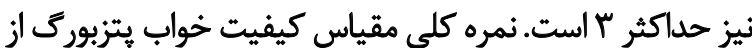

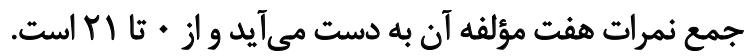

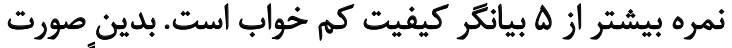

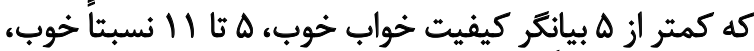

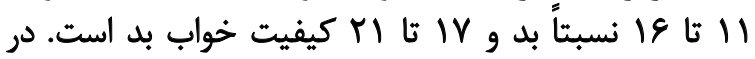
اين مطالعه نمره ه ثا 19 متوسط در نظر ترفته شد. اعتبار

2. The Pittsburgh Sleep Quality Index (PSQI)

3. General Health Questionnaire (GHQ-12)

4. Vaux Social Support Questionnaire
در مطالعات إيبدميولوزيك نيز نشان داده شده است كه بيش

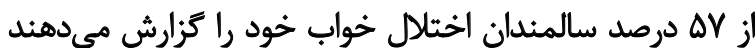

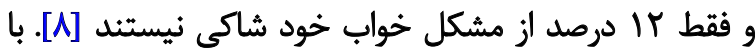

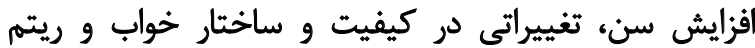

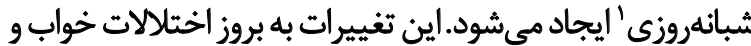

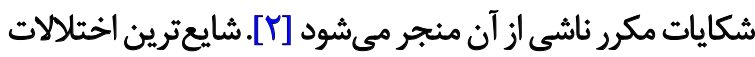

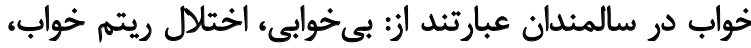

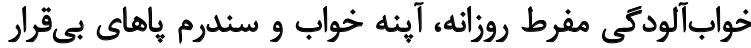

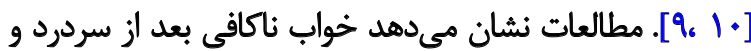

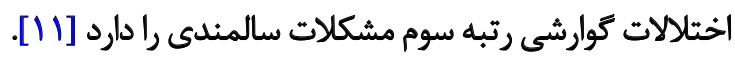
خواب ناكافى با افزايش بيمارى، نرخ بسترىشدن درئ در افراد

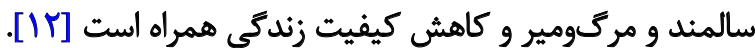

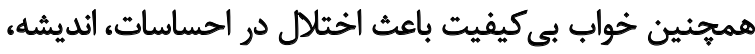

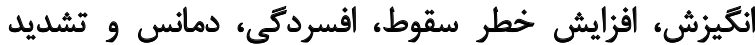

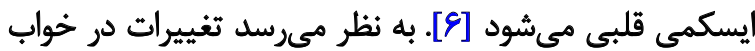

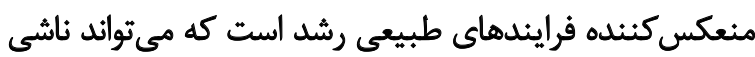

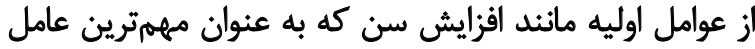

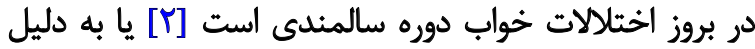

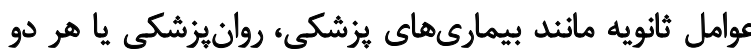

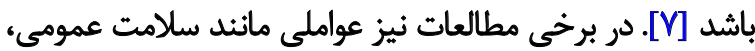

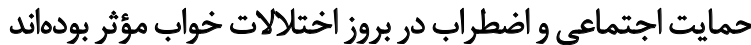

$\left[r_{6} 11.1 \%-10\right]$

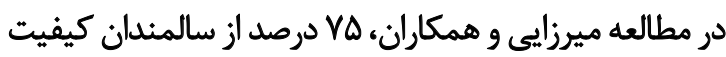

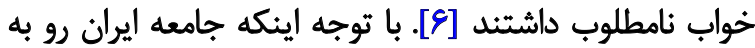

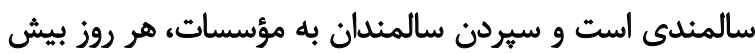

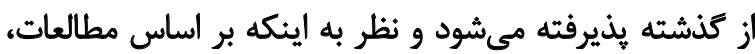

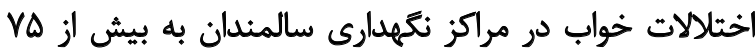

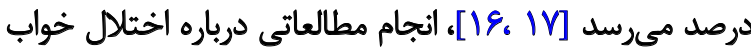

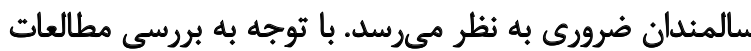

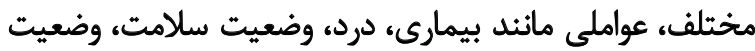

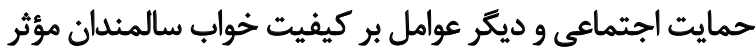

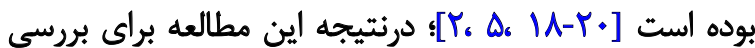

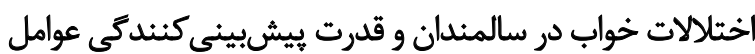
مذكور در بروز اختلالت خواب انجام يذيرفت

از سوى ديكر تاكنون كمتر مطالعهاى به برائ برسى

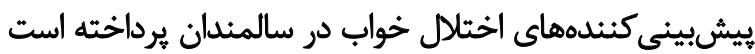

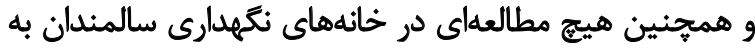

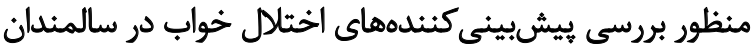

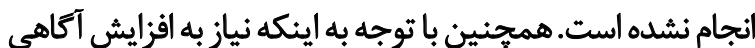

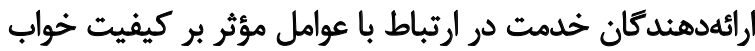

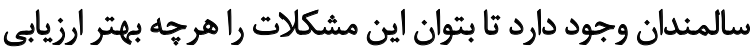


ابراهيمى قوام در ثرؤهش خود هايايى و رواييى اين ابزار را

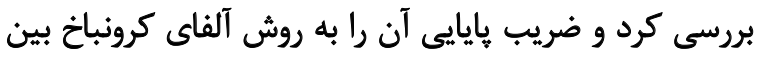

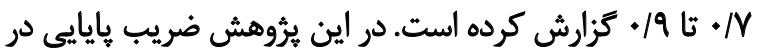

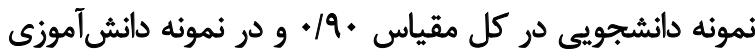

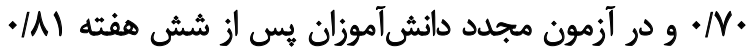

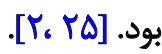

\section{يرسش نامه درد زانوى وومك}

خاين يرسشنامه حاوى Tf سؤال در سه زيرمقياس درد،

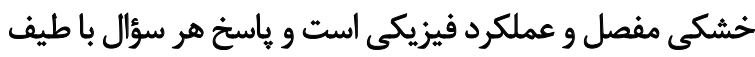

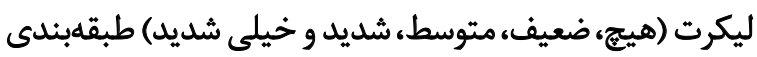

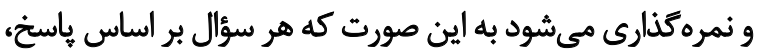

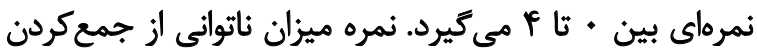

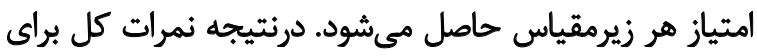

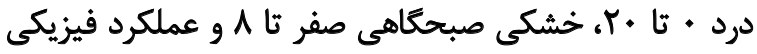

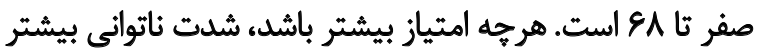

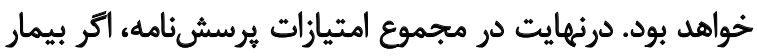

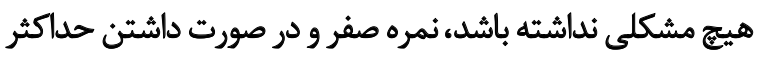

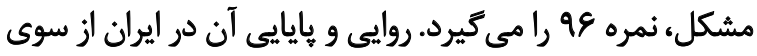

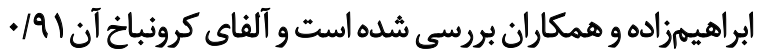

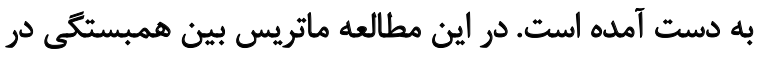

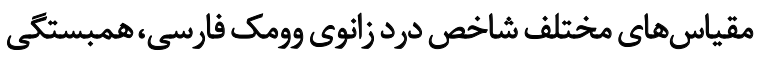

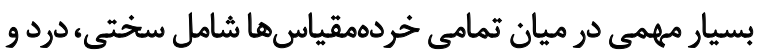

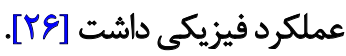

فهرست مشخصات جمعيتشناختى شامل موراد زير است:

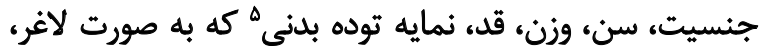

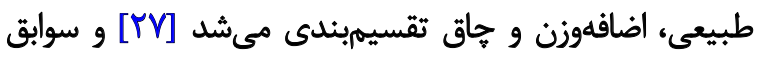

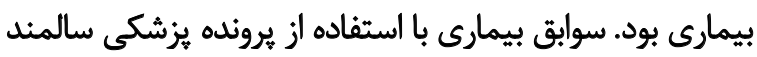

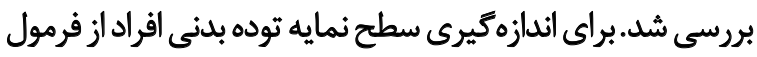

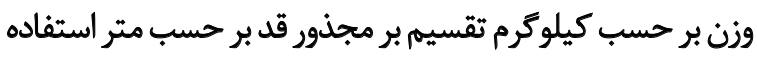

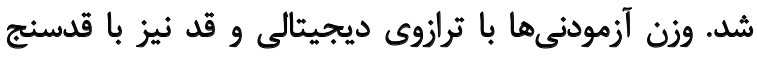

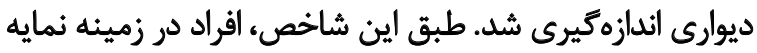

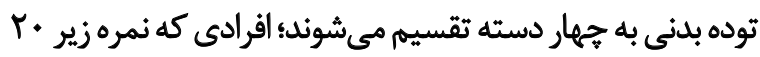

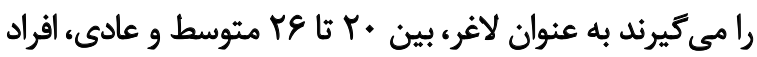

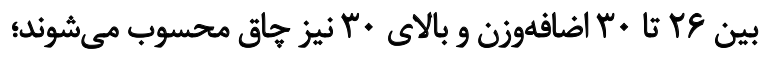
يرسشكر يرسش نامهها از طريق مصاحبه با سالمندان تكميل كرد.

يس از جمعآورى يرسش نامهها، دادهها وارد نسخه بَ بـ نرمافزار

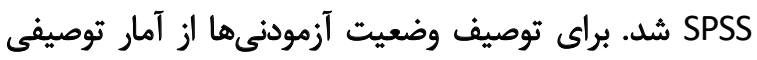

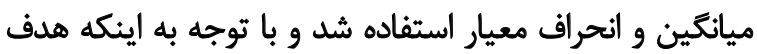

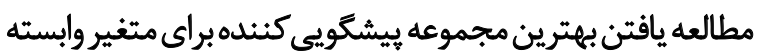

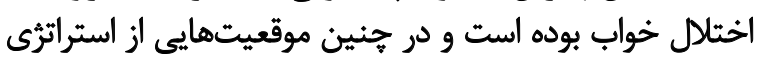

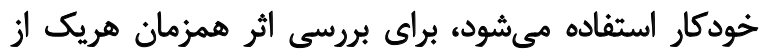

5. Body Mass Index (BMI)

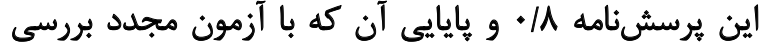

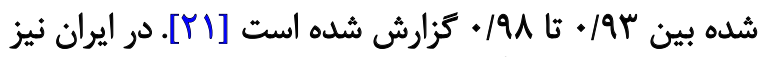

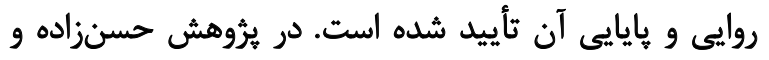

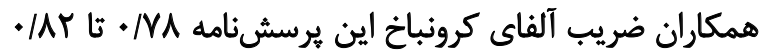

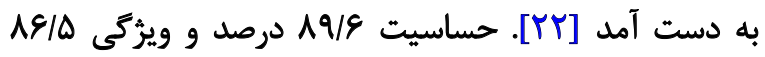

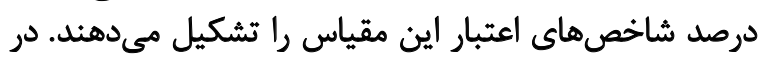

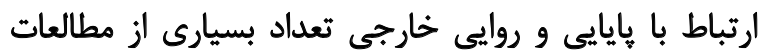

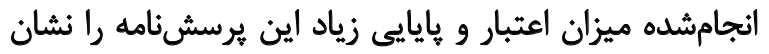

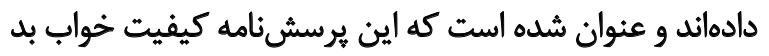

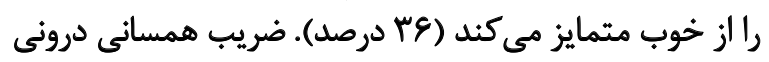

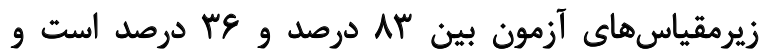

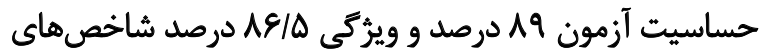

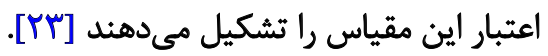

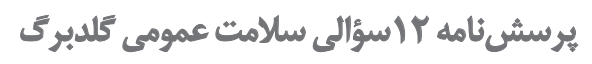

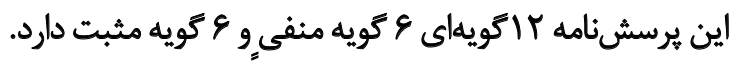

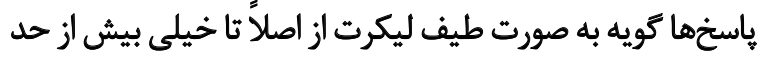

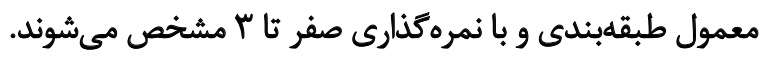

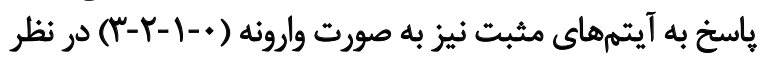

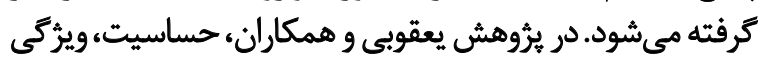

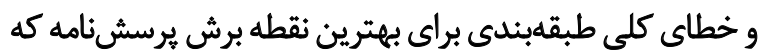

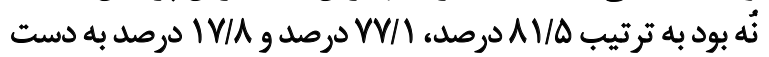

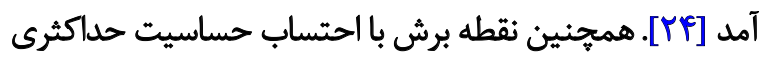

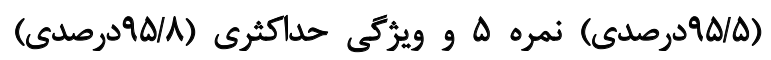

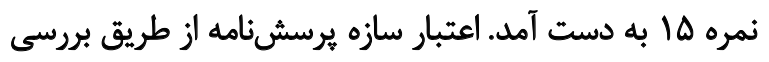

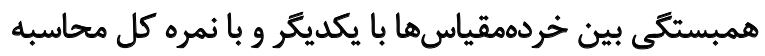

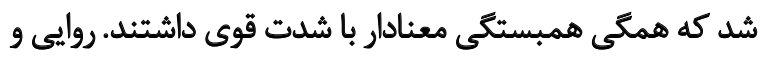

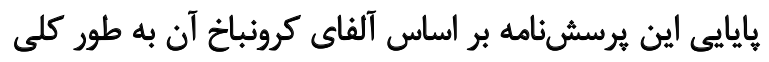

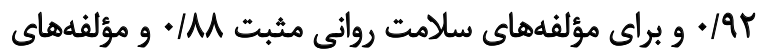

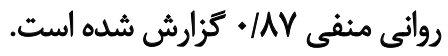

برسش نامه حمايث اجثماعى ادراكشده واكس

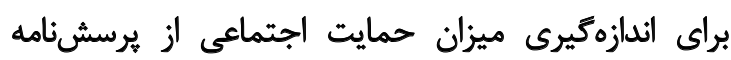

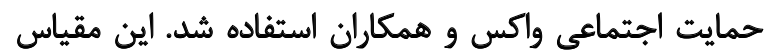

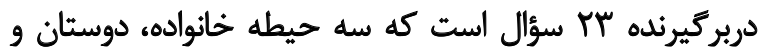

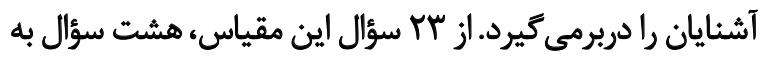

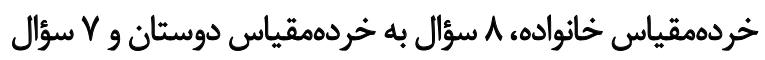

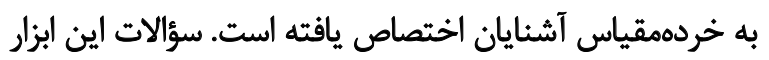

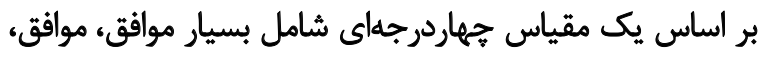

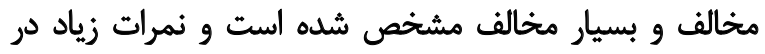

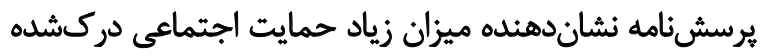

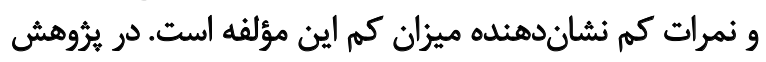

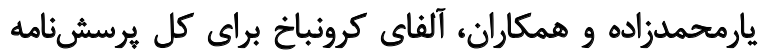
|AF 
برابر

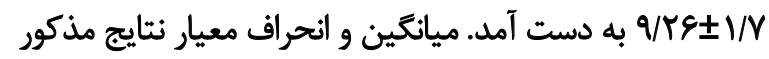

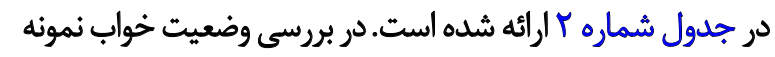

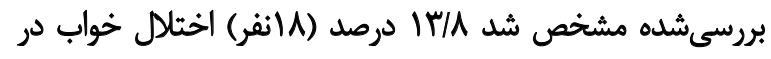

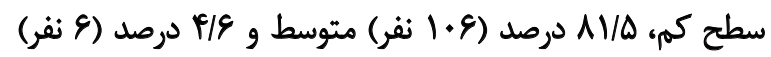
سطح اختلال خواب شديد داشتند (جدول شماره ب).

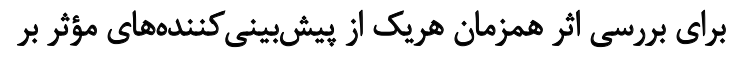

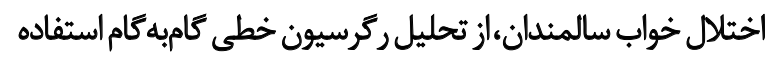

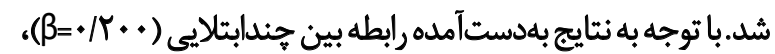

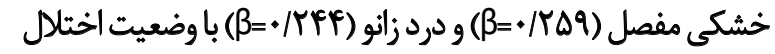

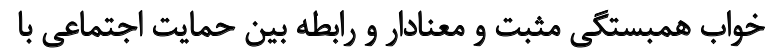

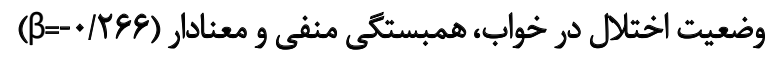

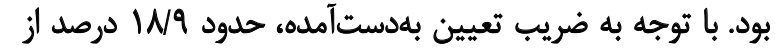

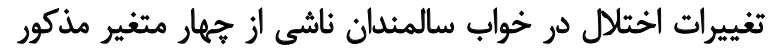

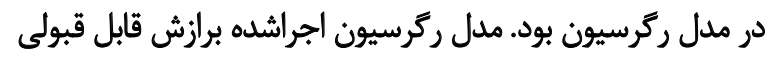

ريشيشينى كنندهاى مؤثر بر اختلال خواب سالمندان از تحليل

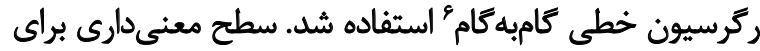

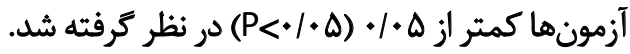

يافتهها

نتايج حاصل از مطالعه نشان داد از ميان · با نفر از افراد تحت

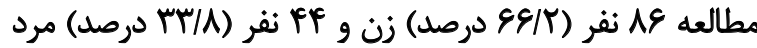

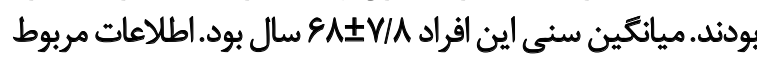
به متغييرهاى فردى در جدول شماره إلرائه شده است.

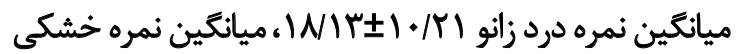
مفصل ميانكين نمره درد

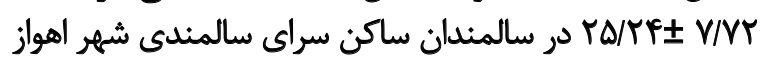

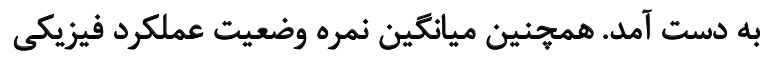

6. Stepwise

جدول ا. توزيع فراوانى متغيرهاى فردى

\begin{tabular}{|c|c|c|c|}
\hline درصد & فراوانى & & \\
\hline $\operatorname{sel}$ & $N$ & زن & \multirow{2}{*}{ جنسيت } \\
\hline$\pi / \Lambda$ & pr & مرد & \\
\hline$r / \Lambda$ & $\Delta$ & لاغر & \multirow{4}{*}{ نمايه توده بلنى } \\
\hline$r a / r$ & A) & عادى & \\
\hline$\pi / \pi$ & Pr & اضافهوزن & \\
\hline$I r / r$ & 19 & جاق & \\
\hline.$/ 1$ & 1 & ندارد & \multirow{12}{*}{ بيمارى } \\
\hline$r / r$ & $r$ & قلبى & \\
\hline$\Delta / \varphi$ & 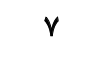 & ديابت & \\
\hline $8 / 9$ & 9 & افسردكى & \\
\hline$+/ 1$ & 1 & يوكى استخوان & \\
\hline$r / \Lambda$ & $\Delta$ & فشارخون بالا & \\
\hline$\varphi / q$ & 8 & اضطراب & \\
\hline$\Delta / f$ & $v$ & هاركينسون & \\
\hline $19 / 9$ & rr & هربى خون بالا & \\
\hline WA & $r f$ & كليوى & \\
\hline $18 / 9$ & rr & ريوى & \\
\hline IW/ & rr & استخوان و مفصل & \\
\hline
\end{tabular}


جدول ז. ميانكين و انحراف معيار نمرات اختّلال خواب، سلامت عمومى، عملكرد فيزيكى، درد زانو وخشكى مفصل

\begin{tabular}{|c|c|c|}
\hline انحراف هعيار & مياتعين & متغير \\
\hline $1 . / M$ & WIr & مرد زانو \\
\hline$r / M$ & fhr & خشكى مفصل \\
\hline $10 / 19$ & ( & عملكرد فيزيكى \\
\hline$V / M r$ & ra/TH & حمايت اجتماعى دركشده \\
\hline $1 / V$ & VTE & سلامت عمومى \\
\hline
\end{tabular}

جدول ب. توزيع فراوانى اختلال خواب در افراد تحت مطالعه

\begin{tabular}{|c|c|c|c|}
\hline درصد & فراوائى & & \\
\hline $\mid r / A$ & $M$ & سطع بايين & \\
\hline$A 1 / \Delta$ & $1+8$ & سطح متوسط & اختلال خواب \\
\hline$r / 8$ & 8 & سطح شديد & \\
\hline
\end{tabular}

2

و حدود W/ درصد از تغييرات اين متغير راتبيين كردند.

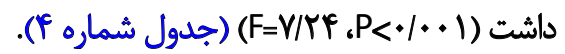

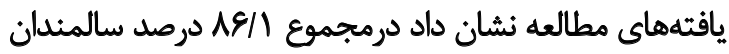

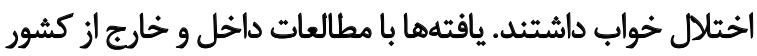
همسو است؛ به طورى كه نتايج مطالعه مقصودى و همكاران

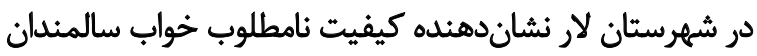

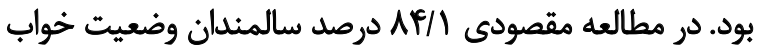
نامطلوب داشتند و در اين ميان كيفيت خواب افرادي ماد ساكن سراي

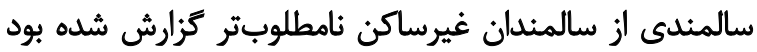

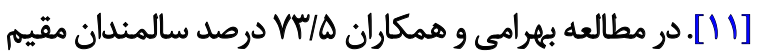

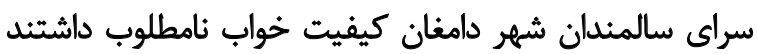

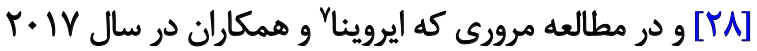

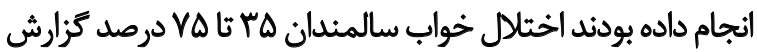

شده است [rq].

7. Irwina

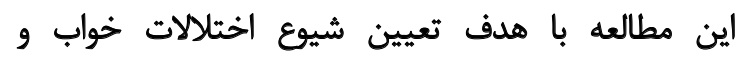

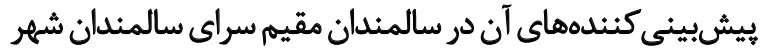

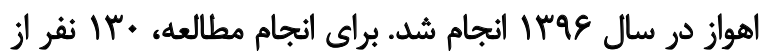

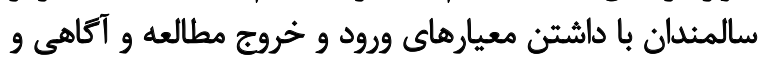

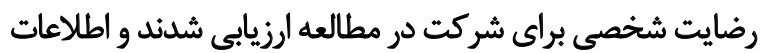

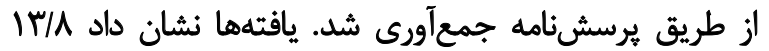

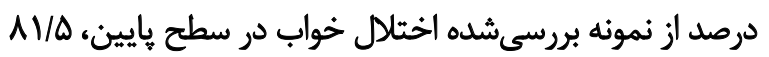

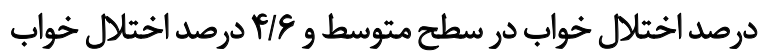

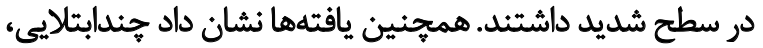

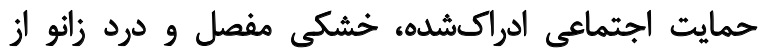

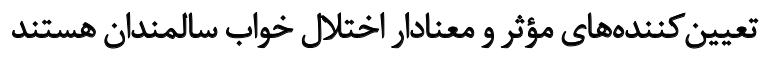

جدول †. نتايج تحليل ركرسيون عوامل هؤثر بر اختلال خواب سالمندان

\begin{tabular}{|c|c|c|c|c|c|}
\hline $\mathbf{P}$ & $\mathrm{T}$ & $\beta$ & S.E & B & متغير ييشبين \\
\hline $.10+1$ & d/Ar & - & 1/1AD & $1 . / 18$ & مقلار ثابت \\
\hline .1. YA & $r / T V$ & $* / r \cdot$. & $.11 \cdot 9$ & - MPV & 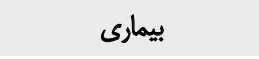 \\
\hline..$+ r$ & $\mathrm{r} / * \Lambda$ &.$- /$ TeE & $.1+4$ &.$- / 1$ ro & حمايت اجتماعى دركشده \\
\hline$\%+1$ & $r / M$ & - RQQ &.$/ 18$ &.$/ 4 \pi r$ & خشكى مفصل \\
\hline $.1+14$ & $r / \Delta r$ & . MTF & $1 \cdot m f$ & $+1+18$ & دردزانو \\
\hline
\end{tabular}

$R=\cdot / 4 r \Delta, R^{2}=* 11 \wedge 9$ ، $F=V / T F, P<\cdot 1 \cdot \circ 1$ 
مبتلا به درد مزمن دريافت مي كند، از طرف خانواده نزديك خود

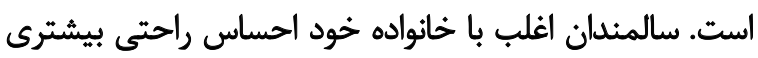

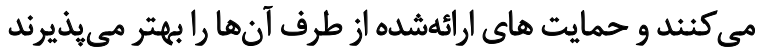

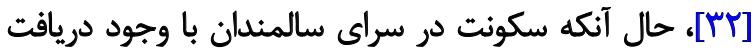

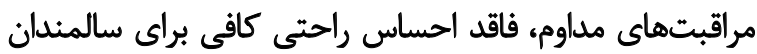

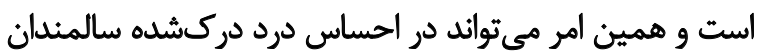

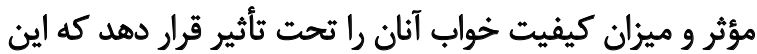

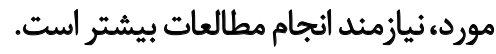

درد به طور كلى موجب كاهش كيفيت زندكى در افراد مي شيود

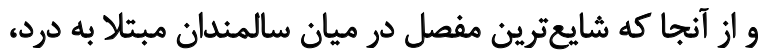

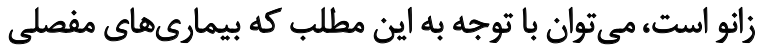

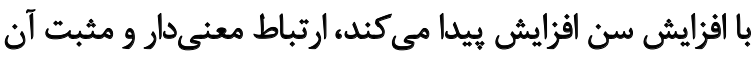

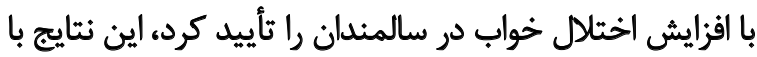

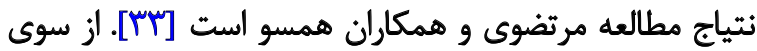

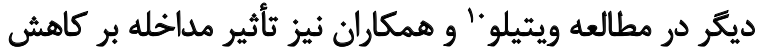

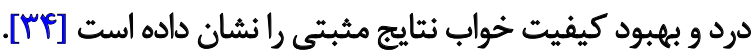

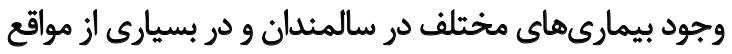

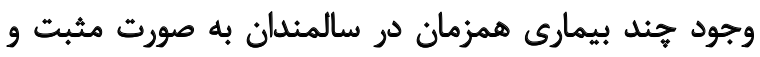

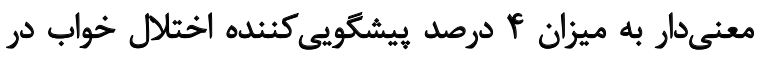

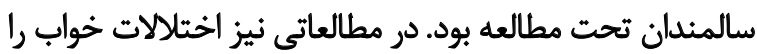

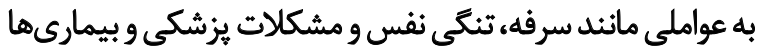

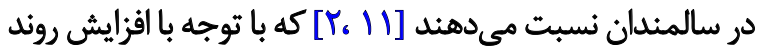

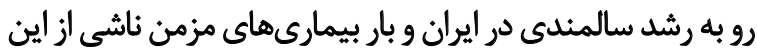

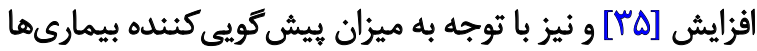

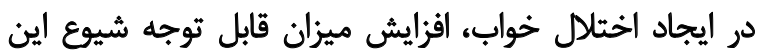
اختلالات در آينده را بيش رواب، افزيش خواهيم داشت

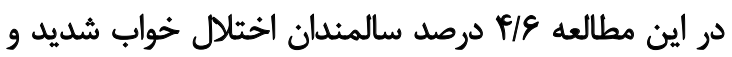

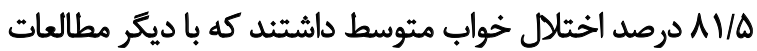

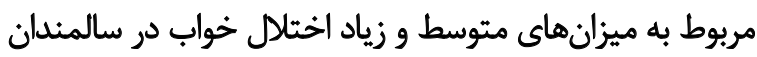

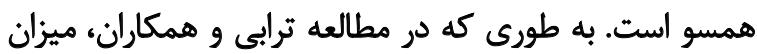

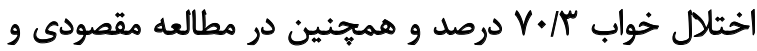

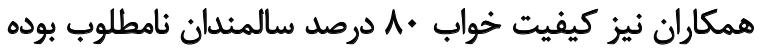

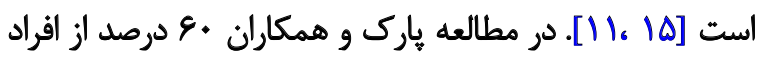

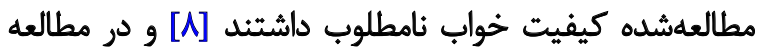

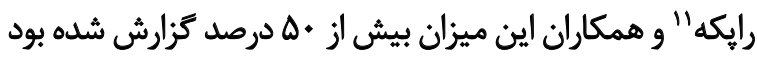
[عץ] كه با نتايج اين مطالعه همسو است.

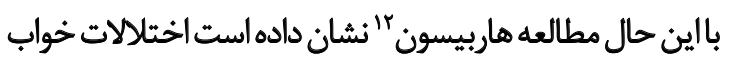

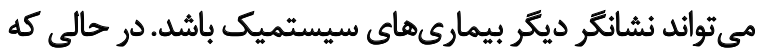

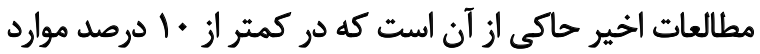

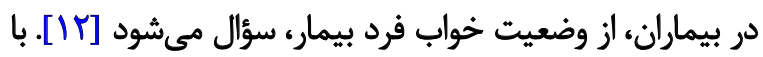

اين مطالعه نشان داد حمايت اجتماعى دركشده، هندئدابتلايى

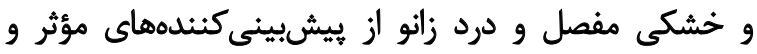

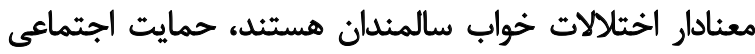

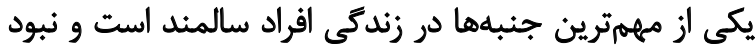

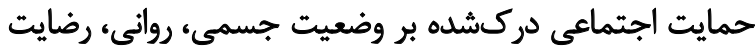

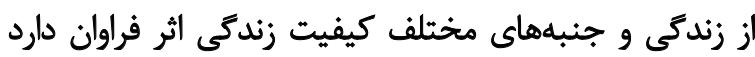

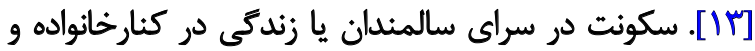

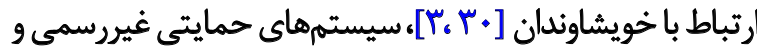

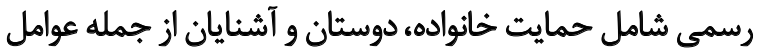

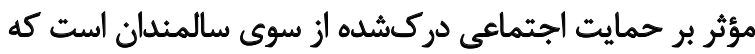

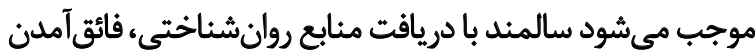

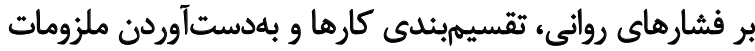

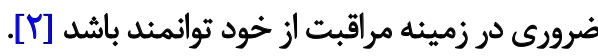

در اين مطالعه ميانگين حمايت اجتماعى دركشده از إز

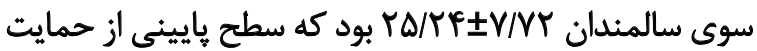

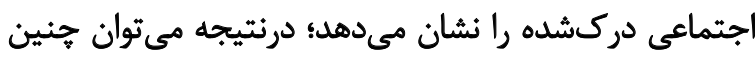

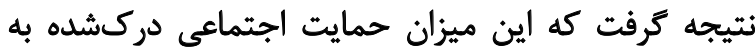

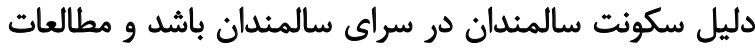

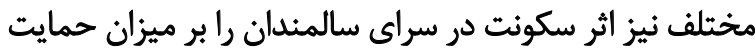

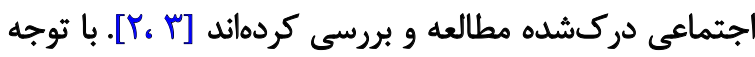

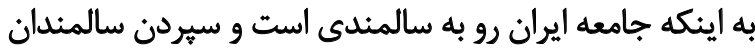

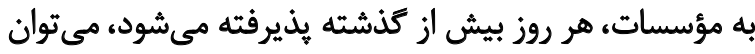

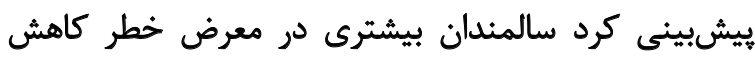

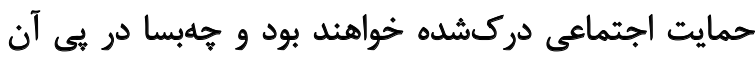

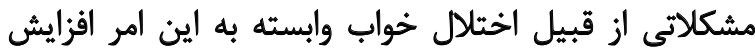

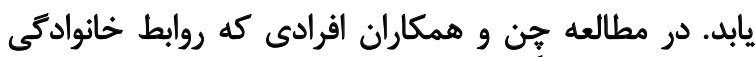

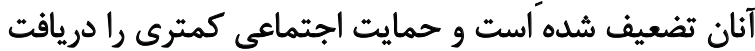

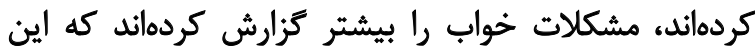

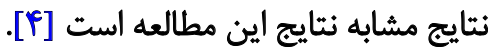

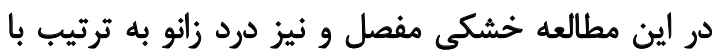

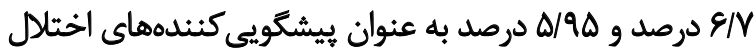

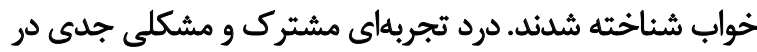

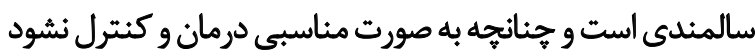

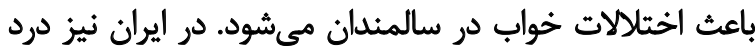

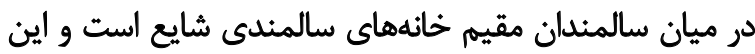

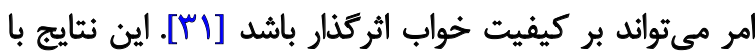

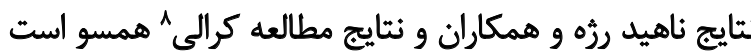

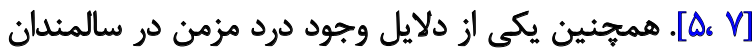

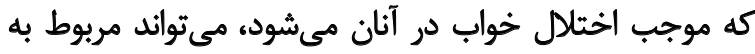

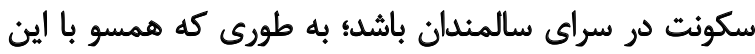

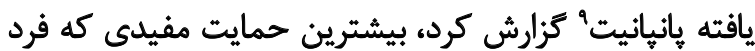

\section{Crowley}

9. Panpanit 
اخلاق IR.AJUMS.REC1394.142 در دانشكاه علوم يزشكى

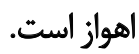

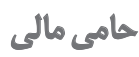

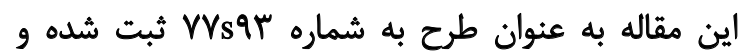

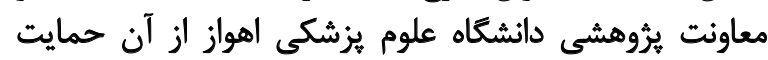

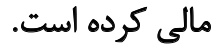

$$
\text { مشاركت ثويسندكان }
$$

مفهومسازى: شهاب بايیى، زينب كريمى، يروين شهرى؛ تحقيق بريق

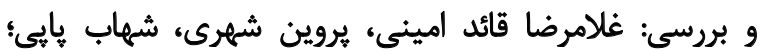

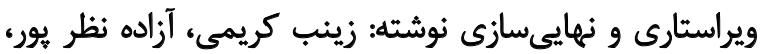

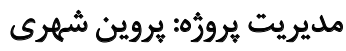

$$
\text { تعارض مثافع }
$$

وجنا به اظهار نويسندكان، در اين مقاله هيجّونه تعارض منافعى

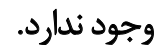

$$
\text { تشكر و قدردانى }
$$

نويسندكان بر خود لازم مي النئد مراتب تشكر و قدردانى

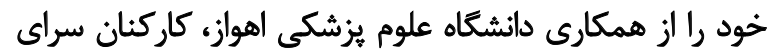

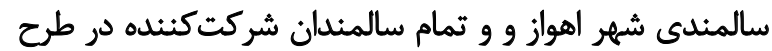
اعلام كنيند. - المير
توجه به نتايج مطالعه و نيز ميزان قابل توجه اختلالات خواب

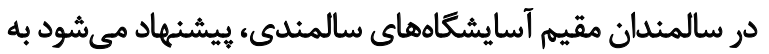

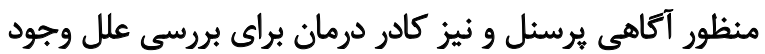

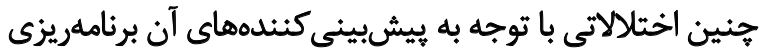

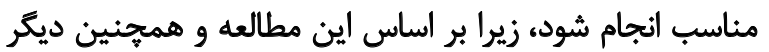

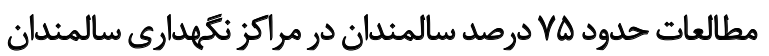
دجار اختلالات خواب هستند [19، IV]

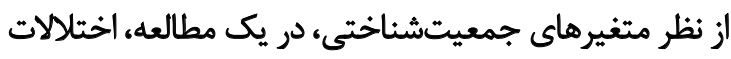

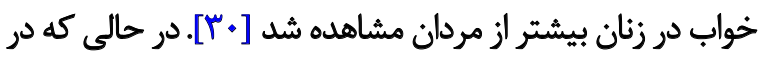

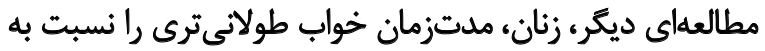

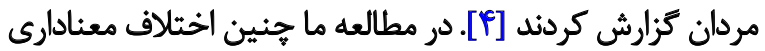

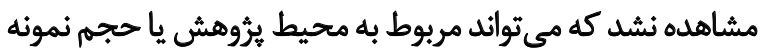

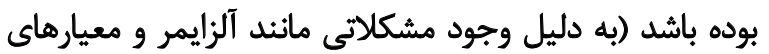

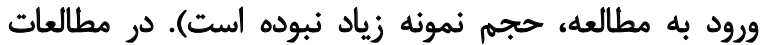

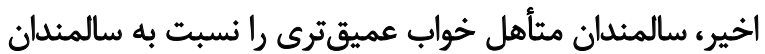

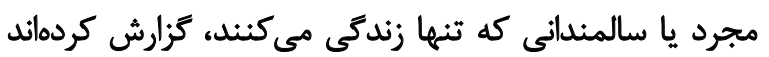

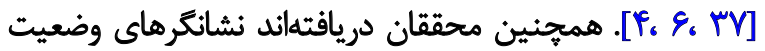

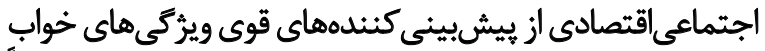

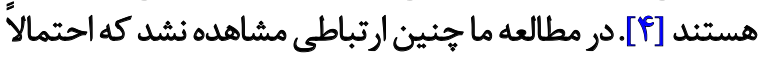

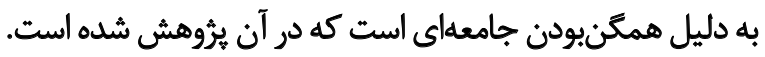

\section{نتيجلكَيرى نههايى}

نتايج مطالعه نشان داد / 19/ درصد سالمندان اختلال خواب

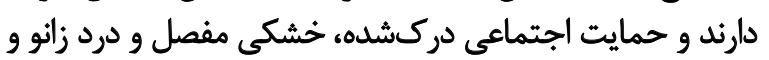

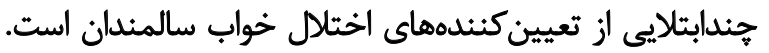

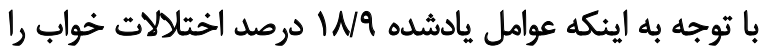

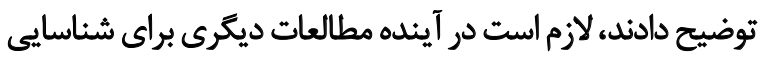

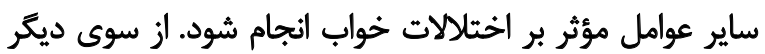

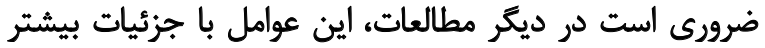

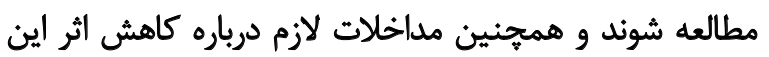

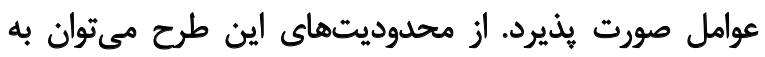

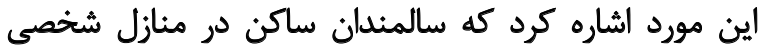

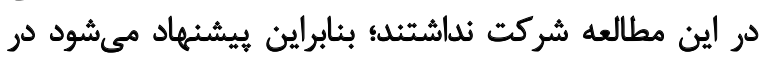

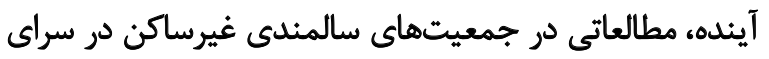

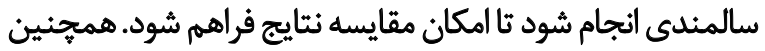

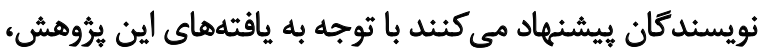

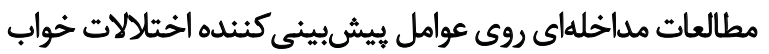

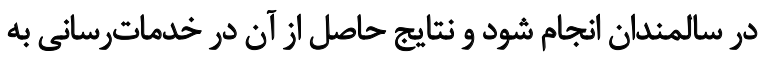
اين قشر از جامعه، بلويرُه در خانههاي سالمندى بـ به كار برده شود.

مالاحظات اخلاقى يبروى ائز اصول اخلاق يثروهش اين مطالعه حاصل طرح تحقيقاتى با كد طرح rVو9 و كد 


\section{References}

[1] Salmabadi M, Rajabi MJ, Safara M, Kokabi R. [Effectiveness of training the review of lfe on life satisfaction and sense of coherence middle-aged women and elderly nursing home residents in Qazvin (Persian)]. Iranian Journal of Ageing. 2018; 13(2):198-209. [DOI:10.32598/sija.13.2.198]

[2] Poursharifi H, Farahmand SM. [The relationship between social support and sleep quality with general health in residing nursing house elderly (Persian)]. Journal of Aging Psychology. 2015; 1(1):31-9.

[3] Momeni K, Karimi H. [Comparison of mental health between elderly admitted in sanitarium with elderly in sited in personal home (Persian)]. Journal of Kermanshah University of Medical Sciences. 2011; 14(4):328-35.

[4] Chen JH, Lauderdale DS, Waite LJ. Social participation and older adults' sleep. Social Science \& Medicine. 2016; 149:164-73. [DOI:10.1016/j.socscimed.2015.11.045] [PMID] [PMCID]

[5] Rejeh N, Heravi KM, Foroughan M. [An exploration into the lived experiences of the hospitalized older women of sleep phenomenon and its disturbance: A qualitative study (Persian)]. Scientific-Research Journal of Shahed University. 2010; 17(86):1-13.

[6] Mirzaei M, Gholamrezaei E, Bidaki R, Fallahzadeh H, Ravaei J. [Quality of sleep and methods of management of sleep disorders in elderly of Yazd city in 2016 (Persian)]. Journal of Shahid Sadoughi University of Medical Sciences. 2017; 25(6):467-75.

[7] Crowley K. Sleep and sleep disorders in older adults. Neuropsychology Review. 2011; 21(1):41-53. [DOI:10.1007/s11065-0109154-6] [PMID]

[8] Park JH, Yoo MS, Bae SH. Prevalence and predictors of poor sleep quality in Korean older adults. International Journal of Nursing Practice. 2013; 19(2):116-23. [DOI:10.1111/ijn.12047] [PMID]

[9] Neikrug AB, Ancoli-Israel S. Sleep disorders in the older adult-a mini-review. Gerontology. 2010; 56(2):181-9. [DOI:10.1159/000236900] [PMID] [PMCID]

[10] Malakouti SK, Foroughan M, Nojomi M, Ghalebandi MF, Zandi T. Sleep patterns, sleep disturbances and sleepiness in retired Iranian elders. International Journal of Geriatric Psychiatry. 2009; 24(11):1201-8. [DOI:10.1002/gps.2246] [PMID]

[11] Maghsoudi A, Dindarloo S, Jamali T, Ghaed S, Rastgoo Z, Azgomi SH. [Comparison of sleep quality and general health in elderly individuals living in their houses and nursing homes (Persian)]. Sadra Medical Sciences Journal. 2017; 4(3):161-72.

[12] Harbison J. Sleep disorders in older people. Age and ageing. 2002; 31(suppl 2):6-9. [DOI:10.1093/ageing/31.suppl_2.6]

[13] Farhadi A, Obeidavi Z, Movahedi Y, Rahimi M, Mobasher A. [The comparison and relationship of social support and stress with life satisfaction among the elderly of Khorramabad city in 2013 (Persian)]. Yafte. 2015; 17(2):14-22.

[14] Hosseini M, Dakhteh Hm, Yaghmaei F, Alavi Mh. [Correlation of social support and health in an elderly population in Iran (Persian)]. Advances in Nursing \& Midwifery. 2011; 21(73):25-30.

[15] Torabi S, Shahriari L, Zahedi R, Rahmanian S, Rahmanian K. [A survey the prevalence of sleep disorders and their management in the elderly in Jahrom City, 2008 (Persian)]. Journal of Jahrom University of Medical Sciences. 2013; 10(4):35-41.

[16] Ohayon M, Lemoine P. Sleep and insomnia markers in the general population. L'Encephale. 2004; 30(2):135-40. [DOI:10.1016/ S0013-7006(04)95423-1]

[17] Gentili A, Weiner D, Kuchibhatla M, Edinger J. Factors that disturb sleep in nursing home residents. Aging Clinical and Experimental Research. 1997; 9(3):207-13. [DOI:10.1007/BF03340151]

[18] Freitas D, Simoes L , Dutra S, Oliveira N, Clares S, Cortopassi F, Et Al. Sleep Quality In Long-Living Brazilians. Indian Journal of Applied Research. 2018; 7(4).611-14.

[19] Park NC. AB021. Management of Sleep Disorder in Elderly Patients With Urological Disease. Translational Andrology and Urology. 2017; 6(Suppl 3).207-208. [DOI: 10.21037/tau.2017.s021]

[20] Chiang GS, Sim BL, Lee JJ, Quah JH. Determinants of poor sleep quality in elderly patients with diabetes mellitus, hyperlipidemia and hypertension in Singapore. Primary Health Care Research \& Development. 2018; 19(6):610-5. [DOI:10.1017/ S146342361800018X]

[21] Ancoli-Israel S. Sleep problems in older adults: Putting myths to bed. Geriatrics. 1997; 52(1):20-30. [PMID]

[22] Hasanzadeh M, Alavi Knk, Ghalehbandi M, Gharaei B, Sadeghikia A. [Sleep quality in Iranian drivers recognized as responsible for severe road accidents(Persian)]. 2008; 6(2):97-107.

[23] Afkham Ebrahimi A, Ghale Bandi, M, Salehi, M, Kafian Tafti, A, Vakili, Y, Akhlaghi Farsi, E. [Sleep parameters and the factors affecting the quality of sleep in patients attending selected clinics of Rasoul-e-Akram hospital (Persian)]. Razi Journal of Medical Sciences. 2008; 15:31-38.

[24] Yaghubi H, Karimi M, Omidi A, Barooti E, Abedi M. [Validity and factor structure of the General Health Questionnaire (GHQ12) in university students (Persian)]. International Journal of Behavioral Sciences. 2012; 6(2):153-60.

[25] Yarmohammad Zadeh P, Feyzollahi Z. Determining the relationship between social support, academic motivation and academic self-efficacy of high school students in Tabriz and Azarshahr. Journal of Applied Sociology. 2014; 27(61):157-69. [DOI:10.22108/JAS.2016.20487]

[26] Ebrahimzadeh MH, Makhmalbaf H, Birjandinejad A, Keshtan FG, Hoseini HA, Mazloumi SM. The Western Ontario and McMaster universities Osteoarthritis Index (WOMAC) in Persian speaking patients with knee osteoarthritis. Archives of Bone and Joint Surgery. 2014; 2(1):57-62. [PMID] [PMCID]

[27] khezry A, Arab Ameri E, Hemayattalab R. [The Comparison of response time between active and non-active elderly at different levels of body mass index (Persian)]. Journal of Behavioral Movement and Psychology of Exercise. 2012; 8(1):597-605.

[28] Bahrami M, Dehdashti A, Karami M. [A survey on sleep quality in elderly people living in a nursing home in Damghan city in 2017: A short report (Persian)]. Journal of Rafsanjan University of Medical Sciences. 2017; 16(6):581-90

[29] Irwina AS, Amita A, Ifa N. Quality of sleep on the elderly: Asystematic review; 2017

[30] Sheikhy L, karami Mattin B, chupani J, Hookari S, fallah B. [Evaluation the status of sleep quality in elderly people in Kermanshah city (Persian)]. Rehabilitation Medicine. 2015; 3(4):81-8. 
[31] Shirazi M, Manoochehri H, Zagheri TM, Zayeri F, Alipour V. Explaining of chronic pain management process in older people: A grounded theory study (Persian)]. Medical-Surgical Nursing Journal. 2016; 4(4):1-11.

[32] Panpanit L. [Striving to maintain well-being: Self-management of chronic pain by elderly people living in rural communities in North-East Thailand (Thai)]. [ PhD. dissertation]. Thailand: Victoria University; 2012.

[33] Mortazavi H, Pakniyat A, Ganji R, Armat M, Tabatabaeichehr $\mathrm{M}$, Saadati $\mathrm{H}$. [The Effect of self-management education program on disability of elderly patients with knee osteoarthritis referring to elderly care clinic of Imam Reza (AS) Treatment Center in Shiraz, 2015-2106 (Persian)]. Journal of North Khorasan University of Medical Sciences. 2017; 8(3):461-70. [DOI:10.18869/acadpub. jnkums.8.3.461]

[34] Vitiello MV, Rybarczyk B, Von Korff M, Stepanski EJ. Cognitive behavioral therapy for insomnia improves sleep and decreases pain in older adults with co-morbid insomnia and osteoarthritis. Journal of Clinical Sleep Medicine. 2009; 5(4):355-62. [PMID] [PMCID]

[35] Karimi Z, Majlesi F, Tol A, Rahimi Foroushani A, Sahaf R, Ali $\mathrm{Gol} \mathrm{M}$, et al. [The effect of educational intervention on the promotion of physical activities of the elderly men in Qom City: Application of Trans-Theoretical Model (Persian)]. Iranian Journal of Ageing. 2015; 10(3):182-91.

[36] Roepke SK, Ancoli-Israel S. Sleep disorders in the elderly. Indian Journal of Medical Research .2010; 131:302-10. [PMID]

[37] Izadi F, Adib Hajbagheri M, Kafaei M. [Determining disturbing factors of sleep quality among hospitalized elderly patients in Kashan Hospitals, Iran 2009 (Persian)]. Journal of Shahid Sadoughi University of Medical Sciences. 2013; 20(6):688-98. 